\title{
metabolites
}

ISSN 2218-1989

www.mdpi.com/journal/metabolites/

Article

\section{Metabolomic Analysis of Fission Yeast at the Onset of Nitrogen Starvation}

\author{
Kenichi Sajiki $^{\dagger} *$, Tomáš Pluskal ${ }^{\dagger}$, Mizuki Shimanuki ${ }^{\star}$ and Mitsuhiro Yanagida * \\ G0 Cell Unit, Okinawa Institute of Science and Technology Graduate University (OIST), \\ 1919-1 Tancha, Onna-son, Okinawa 904-0495, Japan; E-Mails: pluskal@oist.jp (T.P.); \\ mizuki.shimanuki@oist.jp (M.S.)
}

$\dagger$ These authors contributed equally to this work.

* Current address: Biology Resources Section, Okinawa Institute of Science and Technology Graduate University (OIST), 1919-1 Tancha, Onna-son, Okinawa 904-0495, Japan.

* Authors to whom correspondence should be addressed; E-Mails: sajiki@oist.jp (K.S.); myanagid@gmail.com (M.Y.); Tel.: +81-98-966-8858 (K.S.); +81-98-966-8658 (M.Y.); Fax: +81-98-966-2890.

Received: 8 November 2013; in revised form: 3 December 2013 / Accepted: 6 December 2013 / Published: 13 December 2013

\begin{abstract}
Microorganisms naturally respond to changes in nutritional conditions by adjusting their morphology and physiology. The cellular response of the fission yeast $S$. pombe to nitrogen starvation has been extensively studied. Here, we report time course metabolomic analysis during one hour immediately after nitrogen starvation, prior to any visible changes in cell morphology except for a tiny increase of cell length per division cycle. We semi-quantitatively measured 75 distinct metabolites, $60 \%$ of which changed their level over 2-fold. The most significant changes occurred during the first $15 \mathrm{~min}$, when trehalose, 2-oxoglutarate, and succinate increased, while purine biosynthesis intermediates rapidly diminished. At 30-60 min, free amino acids decreased, although several modified amino acids - including hercynylcysteine sulfoxide, a precursor to ergothioneine - accumulated. Most high-energy metabolites such as ATP, S-adenosyl-methionine or $\mathrm{NAD}^{+}$remained stable during the whole time course. Very rapid metabolic changes such as the shut-off of purine biosynthesis and the rise of 2-oxoglutarate and succinate can be explained by the depletion of $\mathrm{NH}_{4} \mathrm{Cl}$. The changes in the levels of key metabolites, particularly 2-oxoglutarate, might represent an important mechanistic step to trigger subsequent cellular regulations.
\end{abstract}


Keywords: fission yeast; nitrogen starvation; metabolomics; 2-oxoglutarate; trehalose; AICAR; ergothioneine

\section{Introduction}

Microorganisms naturally respond to changes in nutritional conditions by adjusting their morphology and physiology. The fission yeast Schizosaccharomyces pombe, a popular model organism in cell cycle research, exhibits a remarkable phenotype under nitrogen starvation (hereafter N-starvation). Following the withdrawal of the nitrogen source $\left(\mathrm{NH}_{4} \mathrm{Cl}\right)$ from the culture medium, $S$. pombe cells exit the cell cycle, inhibit cellular growth and enter the G0 phase, thus providing an excellent model to study cellular quiescence [1].

The adaptation to $\mathrm{N}$-starvation occurs in several stages. In the initial stage (within the first $\sim 8 \mathrm{~h}$ ), the cell size is reduced by two subsequent cell divisions, consequently forming small and round cells [2]. Following the two rounds of division, the cell cycle is arrested pre-replicatively in an 'uncommitted' G1 phase [3]. During this period, cells may undergo meiosis and produce spores, providing that a partner of the opposite mating type is available. In the absence of a mating partner, the cells lose the ability to mate after $12 \mathrm{~h}$ and commit to the G0 phase [4]. Within $24 \mathrm{~h}$ of $\mathrm{N}$-starvation, the cellular volume, mRNA and protein content are reduced to about 55, 20, and 50\% of the vegetative cell content, respectively [5]. Fully adapted G0 cells can survive for months in the N-starvation culture medium, exhibiting increased stress resistance [6,7].

To elucidate the molecular mechanisms of the $\mathrm{N}$-starvation effect in $S$. pombe, a number of genetic, transcriptomic and proteomic studies were conducted [2,5,8-11]. We previously reported 33 "super-housekeeping" genes required for N-starvation-induced quiescence [10]. Microarray analysis showed alterations in the transcriptional profiles of almost all genes immediately after N-starvation [8]. About 900 transcripts ( $\sim 19 \%$ of $\sim 4,600$ detected), half of which corresponded to stress-responsive genes, were up- or down-regulated more than 3-fold. Such immense change in gene expression occurred as early as one hour after N-starvation, indicating the molecular events occurring during the first hour might be critical for the cellular adaptation. Kristell et al. reported $>2$-fold alteration of 229 transcripts within 20 min after N-starvation, accompanied by significant changes of chromatin structure in certain genomic regions [9]. Transcription of stress-related genes increased transiently up to $2 \mathrm{~h}$ after $\mathrm{N}$-starvation, followed by a decrease afterwards. On the contrary, transcription of growth-related genes decreased immediately after N-starvation [5]. Certain transcriptional responses, however, occurred only at a later time. For example, the induction of meiosis-related genes regulated by the transcription factor Ste1 1 took place about $3 \mathrm{~h}$ after $\mathrm{N}$-starvation [8]. On the proteome level, $47 \%$ of all detected proteins changed their copy numbers over 2-fold and an extensive shift from growth-related proteins to stress-responsive proteins was observed $24 \mathrm{~h}$ after N-starvation [5].

$\mathrm{N}$-starvation is ultimately a metabolic condition. Therefore, complete understanding of its effect cannot be achieved without studying the intracellular metabolome. In addition, the most immediate response to $\mathrm{N}$-starvation might not be mediated on the transcriptional level, as the transcription/translation machinery responds with an inevitable delay. In this study we carried out metabolomic analysis of 
S. pombe cells during short time course immediately following N-starvation. We focus on the time period of $1 \mathrm{~h}$, before any obvious morphological changes in the cells can be observed. It should be emphasized that the two cell divisions, which represent the key steps in cellular adaptation to N-starvation in $S$. pombe, occur only after this time period. We thus aspire to characterize the direct and immediate metabolic response to $\mathrm{N}$-starvation.

\section{Results and Discussion}

\subsection{Increase in Cell Size and Number Shortly after N-Starvation}

Immediately after the shift to N-starvation, $S$. pombe cells rapidly inhibit their growth (cell length increase), as observed from single cell length measurements (Figure 1A). The length of a N-starved cell increased only about $\sim 1 \mu \mathrm{m}$ per division cycle, considerably less than that of a vegetative cell, about $7 \mu \mathrm{m}$. Such result is consistent with the previously reported swift reduction in growth-related transcripts as well as proteins [5]. Cell division was transiently arrested for 15 min after N-starvation (Figure 1B). After $15 \mathrm{~min}$, the cell number started to increase gradually, although considerably slower than that of vegetative cells. Interestingly, the septation index increased 15 min after $\mathrm{N}$-starvation, indicating delayed cytokinesis (Figure 1C). $60 \mathrm{~min}$ after $\mathrm{N}$-starvation, septation index reached above $20 \%$, while cell number increased only $10 \%$. After $120 \mathrm{~min}$ the cell number increase accelerated and septation index returned to $\sim 13 \%$. Eventually, after the full adaptation to N-starvation $(24 \mathrm{~h})$, septation index became $0 \%$.

Figure 1. (A) Cell length measurement in the $+\mathrm{N}$ or $-\mathrm{N}$ medium. $+\mathrm{N}$ cells were incubated in EMM2; $-\mathrm{N}$ cells were transferred from EMM2 to EMM2-N; (B) Cell number measurement after medium change. Cells incubated initially in EMM2 were transferred to EMM2-N or fresh EMM2 medium at 0 min. The cell concentration was measured and plotted as a fold change against 0 min. DAPI images of cells at $60 \mathrm{~min}$ and $480 \mathrm{~min}$ are shown; (C) Septation index of $S$. pombe cells in asynchronous cell culture after the medium change from EMM2 to EMM2-N.
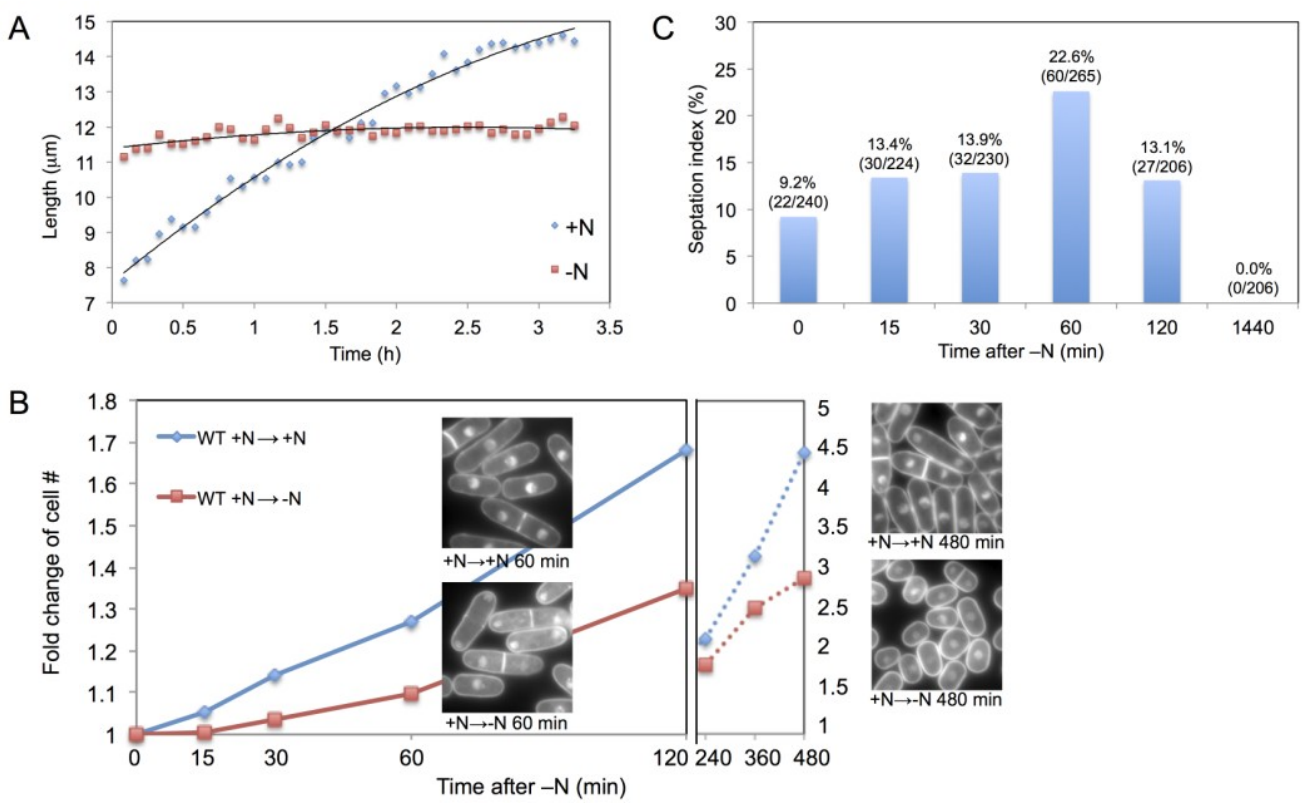


\subsection{Time Course Metabolomic Analysis and Data Reproducibility}

To obtain metabolomic data sets, three independent wild-type $S$. pombe cultures were cultivated at $26{ }^{\circ} \mathrm{C}$ in liquid EMM2 medium to mid-log phase $\left(5 \times 10^{6}\right.$ cells $\left./ \mathrm{mL}\right)$. Cells were collected by vacuum filtration and shifted to EMM2-N medium (EMM2 lacking $\mathrm{NH}_{4} \mathrm{Cl}$ ) to induce $\mathrm{N}$-starvation. Aliquots of $40 \mathrm{~mL}$ were taken from each culture at the time points of 0 (corresponding to the vegetative culture before medium shift), 15, 30 and $60 \mathrm{~min}$. Each aliquot was immediately quenched and used to prepare metabolite extracts. The experiment design is schematized in Figure 2A. Two internal standards, PIPES and HEPES, were spiked into each sample prior to metabolite extraction.

Figure 2. (A) Scheme of the time course metabolome experiment; (B) Principal component analysis (PCA) was conducted for the entire dataset of 75 identified metabolites (Supplementary Table S1) using the SIMCA-P+ software (Umetrics Inc., Umeå, Sweden). A scatter plot of the scores in the first two principal components is shown, with samples color-labeled according to time points; (C) Two normalized peak areas of all metabolites from two different samples taken at the same time point were plotted to demonstrate the data reproducibility; (D) The time course change of the peak areas of 75 metabolites in $\log _{2}$ values was clustered hierarchically by a complete linkage method using the Cluster 3.0 software ver. 1.50 [12] and visualized as a tree view image by the Java TreeView software ver. 1.1.6r2 [13]. (E) The time course change of the peak area of trehalose in cells after N-starvation.
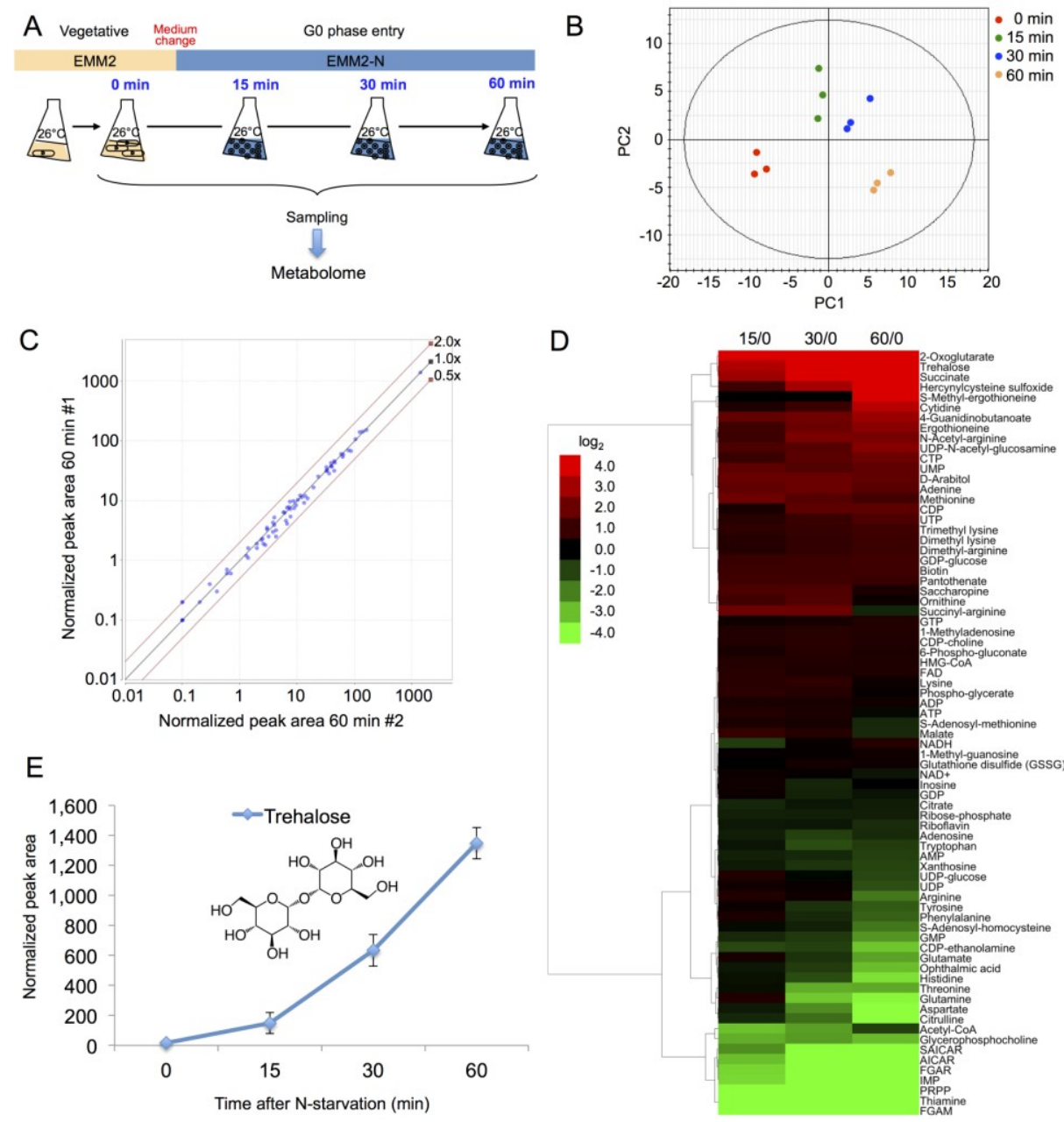
The metabolite extracts were separated by liquid chromatography on the ZIC-pHILIC column (Merck SeQuant, Umeå, Sweden) and measured on the LTQ Orbitrap mass spectrometer (Thermo Fisher Scientific, Waltham, MA, USA) in both positive and negative ionization modes. Raw mass spectra were analyzed by the MZmine 2 software [14]. Over ten thousand distinct peaks were detected in all samples. Principal component analysis calculated from the peak areas of all detected metabolites could clearly differentiate the triplicates of samples obtained at the same time point, as shown in the 2D plot of the first two principal components (Figure 2B).

Among the detected peaks, 75 individual metabolite signals were identified, mainly by comparing their $\mathrm{m} / \mathrm{z}$ values and retention times with authentic pure standards. For 4 compounds, however, pure standards were not available; those were thus identified either by their MS/MS spectra (FGAR, SAICAR, and hercynylcysteine sulfoxide; MS/MS spectra shown in Supplementary Figure S1), or solely by the $\mathrm{m} / \mathrm{z}$ value in the case of FGAM. The peak areas of the identified metabolites were measured and normalized by the peak areas of the spiked internal standards. The reproducibility of metabolite peak areas was very good between the samples prepared independently at the same time point, as shown in Figure 2C.

\subsection{Partial Remodeling of the Cellular Metabolome after N-Starvation}

The time course results of all detected metabolites are summarized in Figure 2D (complete numerical results in Supplementary Table S1). During the $60 \mathrm{~min}, 6$ metabolites (2-oxoglutarate, cytidine, hercynylcysteine sulfoxide, S-methyl-ergothioneine, succinate and trehalose) increased over 10-fold and 11 metabolites (AICAR, aspartate, citruline, FGAM, FGAR, glutamine, histidine, IMP, PRPP, SAICAR and thiamine) decreased over 10-fold. Interestingly, the level of high-energy compounds such as adenosine triphosphate (ATP), S-adenosyl methionine (SAM) or nicotinamide adenine dinucleotide $\left(\mathrm{NAD}^{+}\right)$remained relatively stable during the time course, suggesting the cellular energy pool was not impaired during the initial stage of $\mathrm{N}$-starvation (Figure 2D, center area). Overall, peak areas of 29 of the 75 identified metabolites $(38.7 \%)$ remained within two-fold change in peak area.

A single metabolite showing the most striking accumulation during the time course was trehalose ( $\sim 80$-fold increase after $60 \mathrm{~min}$; Figure $2 \mathrm{E})$. A similar result was previously reported in Saccharomyces cerevisiae [15]. Trehalose is a membrane and protein stabilizer produced by many microorganisms under stress [16]. In $S$. pombe, trehalose biosynthesis pathway starts with the trehalose-phosphate synthase enzyme Tps1. The tps 1 gene belongs to the group of core environmental stress response (CESR) genes, activated under non-specific stress conditions [17]. We have previously reported trehalose accumulation under heat shock [18] and glucose starvation [19]. Apart from the immediate protective and stabilizing function, trehalose might play an important role as a nutrient stock during months-long cellular quiescence, as it can be broken down into two molecules of glucose by the trehalase Ntp1.

\subsection{Rapid Metabolic Response within 15 min}

The changes of metabolites in $15 \mathrm{~min}$ after $\mathrm{N}$-starvation are plotted in Figure 3A. During this interval, 2-oxoglutarate (2OG) and succinate sharply increased. Both are intermediates in the TCA cycle, however the amount of citrate remained rather constant, suggesting this effect might not be 
related to TCA cycle activity. $20 \mathrm{O}$ is required for ammonia assimilation in $S$. pombe by glutamate synthase [20], depletion of ammonia might thus lead to the accumulation of 2OG. The increase in succinate might be a direct consequence of $2 \mathrm{OG}$ accumulation, as $2 \mathrm{OG}$ can be converted to succinate by 2-oxoglutarate oxygenases [21] (Figure 3B). This reaction requires $\mathrm{O}_{2}$ as a cofactor, suggesting that oxygen requirement at the onset of nitrogen starvation might change.

Figure 3. (A) A scatter plot comparing the normalized peak areas of all metabolites at 0 and 15 min after $\mathrm{N}$-starvation (values represent averages of 3 samples); (B) A schema of 2OG-dependent dioxygenase reaction; $(\mathbf{C}-\mathbf{G})$ The time course change of the normalized peak areas of purine biosynthesis intermediates (C), amino acids (D), modified amino acids $(\mathbf{E})$, ergothioneine and related metabolites $(\mathbf{F})$ and glutathione and ophthalmic acid (G) in cells after $\mathrm{N}$-starvation.
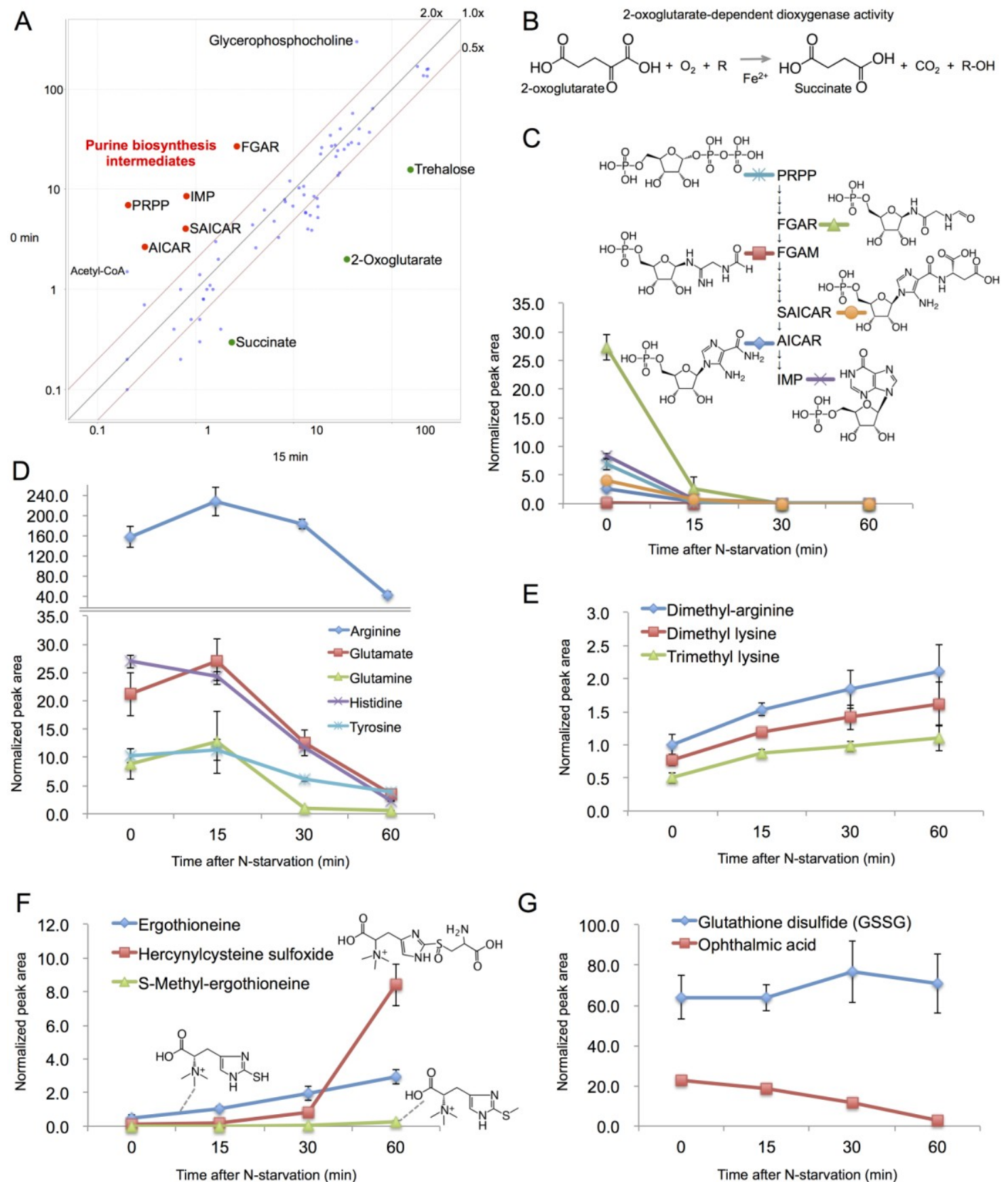

G

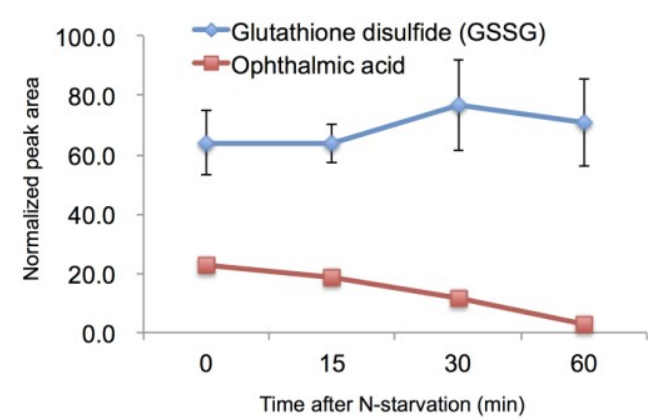


On the other side, PRPP (5-phospho-ribose 1-diphosphate), FGAR (N-formylglycinamide ribonucleotide), FGAM (N-formylglycinamidine ribonucleotide), SAICAR (N-succinocarboxamide-5-aminoimidazole ribonucleotide), AICAR (aminoimidazole-4-carboxamide ribonucleotide) and IMP (inosine-phosphate) decreased dramatically in $15 \mathrm{~min}$ and later became completely undetectable (Figure 3C). These metabolites are intermediates in the purine biosynthesis pathway [22]. In fission yeast, this pathway is mediated by ade genes and the transcripts of ade1, 3, 4, 5, 6, 7, 8, 10 genes were all down-regulated after N-starvation [5]. Following the removal of ammonia from the culture medium, some inhibition of the biosynthesis of nitrogen-rich compounds like purines might be expected. Nevertheless, the speed and scale of the depletion of these compounds is noteworthy, considering there was no visible change in cell morphology at this time point. Contrary to the purine intermediates, glycerophosphocholine decreased $\sim 7$-fold in the first $15 \mathrm{~min}$ (Figure 3A), but its level remained stable afterwards. Glycerophosphocholine is a product of phospholipid catabolism by phospholipases and an important osmolyte [23]. The change in its level might thus reflect the decrease in osmotic pressure due to the missing $\mathrm{NH}_{4} \mathrm{Cl}$ component in the culture medium.

\subsection{Changes in Amino Acid Metabolism after $15 \mathrm{~min}$}

In contrast to the rapid change in metabolite levels in the first $15 \mathrm{~min}$, the following time period was relatively stable, with majority of metabolites staying within 2-fold difference between the consecutive time points (56 of 75 metabolites between 15 and $30 \mathrm{~min}$, and 50 of 75 metabolites between 30 and 60 min, respectively).

The time period between 15 and 60 min after N-starvation was mainly characterized by the changes in amino acid composition. After passing the 15-min time point, all free amino acids sharply declined (Figure 3D). This result might be a direct consequence of the nitrogen source depletion, analogous to the above-described shutdown of purine synthesis. Surprisingly, though, the levels of some modified amino acids, such as methylated lysine and arginine, gradually increased (Figure 3E). Among these was also ergothioneine (Figure 3F), a sulphur-containing derivative of trimethyl histidine, widely believed to act as a physiological antioxidant [24]. Besides ergothioneine itself, we could also detect a sharp increase in hercynylcysteine sulfoxide, known to be a precursor of ergothioneine in Neurospora crassa [25]. Additionally, an S-methyl derivative of ergothioneine was first detected 60 min after $\mathrm{N}$-starvation. No function has so far been reported for this compound.

Two tripeptides were detected in our data set—glutathione (only in its disulfide form, probably due to oxidation during the extraction procedure) and ophthalmic acid. In mouse liver, ophthalmic acid reportedly shares the biosynthesis pathway with glutathione, and exhibits elevated level under oxidative stress when glutathione is depleted [26]. In our experiment, however, the level of ophthalmic acid decreased sharply after N-starvation, while the level of glutathione remained stable (Figure 3G), suggesting a different regulation mechanism might take place in $S$. pombe. 


\section{Experimental Section}

\subsection{Strains and Culture Conditions}

The fission yeast $S$. pombe heterothallic haploid $972 \mathrm{~h}^{-}$wild-type strain [27] was used for the experiments. Cells were incubated in the EMM2 liquid medium [28] at $26{ }^{\circ} \mathrm{C}$ in a water bath with shaking. Cell concentration in the culture was measured by the CDA-500 particle counter (Sysmex, Kobe, Japan). For metabolome analysis, cells were grown to the log phase and at the concentration of $5 \times 10^{6}$ cells $/ \mathrm{mL}, 40 \mathrm{~mL}$ of culture was taken as $0 \mathrm{~min}$ sample. The rest of culture was $\mathrm{N}$-starved by switching the medium with EMM2-N (EMM2 without $\mathrm{NH}_{4} \mathrm{Cl}$ ) following the procedures previously described [7]. After N-starvation, cells were incubated again in the water bath at $26{ }^{\circ} \mathrm{C}$, and after 15, 30, $60 \mathrm{~min}, 40 \mathrm{~mL}$ of culture was taken.

\subsection{Microscopy}

For cell length measurement, time course images were taken by using a DeltaVision Spectris restoration microscope (Applied Precision LLC, Issaquah, WA, USA) with a CH350L CCD camera (Photometrics, Tucson, AZ, USA). Cells were placed on a glass-bottomed culture dish (MatTek, Ashland, OR, USA) and covered with a slip of appropriate agar solid medium. For DAPI staining, cells were fixed with $2 \%$ glutaraldehyde for $10 \mathrm{~min}$ on ice, washed three times with phosphate buffered saline (PBS), and observed under a fluorescence microscope after staining with DAPI $(25 \mu \mathrm{g} / \mathrm{mL})$. Septation index was measured by counting septated cells in about 200 DAPI-stained cells.

\subsection{Metabolome Sample Preparation}

Metabolome samples were prepared by the procedure described previously [18]. Briefly, cells from cultures (40 mL/sample) were collected by vacuum filtration and immediately quenched in $25 \mathrm{~mL}$ of $-40{ }^{\circ} \mathrm{C}$ methanol. Cells were harvested by centrifugation at $-20{ }^{\circ} \mathrm{C}$ and a constant amount of internal standards (10 nmol of HEPES and PIPES) was added to each sample. Cells were disrupted using a Multi-Beads Shocker (Yasui Kikai, Osaka, Japan). Proteins were removed by filtering on an Amicon Ultra 10-kDa cut-off filter (Millipore, Billerica, MA, USA) and samples were concentrated by vacuum evaporation. Finally, each sample was resuspended in $40 \mu \mathrm{L}$ of $50 \%$ acetonitrile and $1 \mu \mathrm{L}$ was used for each LC-MS injection.

\subsection{LC-MS Analysis}

LC-MS data were acquired using a Paradigm MS4 HPLC system (Michrom Bioresources, Auburn, AL, USA) coupled to an LTQ Orbitrap mass spectrometer (Thermo Fisher Scientific, Waltham, MA, USA). LC separation was performed on a ZIC-pHILIC column (Merck SeQuant, Umeå, Sweden; $150 \times 2.1 \mathrm{~mm}, 5 \mu \mathrm{m}$ particle size). Acetonitrile (A) and $10 \mathrm{mM}$ ammonium carbonate buffer, pH 9.3 (B) were used as the mobile phase, with gradient elution from $80 \%$ A to $20 \% \mathrm{~A}$ in $30 \mathrm{~min}$ and $100 \mu \mathrm{L} / \mathrm{min}$ flow rate. Mass spectrometer was operated in full scan mode with the scan range of $100-1,000 \mathrm{~m} / \mathrm{z}$. Each sample was analyzed twice, once in negative and once in positive ionization mode. 


\subsection{LC-MS Data Processing}

Raw LC-MS data were analyzed by the MZmine 2 software [14]. The complete workflow and processing parameters are summarized in Supplementary Table S2.

\section{Conclusions}

In this study we characterized the metabolic response of $S$. pombe to $\mathrm{N}$-starvation during the critical first hour, as schematized in Figure 4. Interestingly, a major remodeling of the cellular metabolome seemed to occur within the first $15 \mathrm{~min}$, while later time points mostly exhibited continuation of the initial trends. The time interval of $15 \mathrm{~min}$ is quite short for gene expression and translation; we might thus suspect that a substantial part of the N-starvation response might be implemented by enzymes already present in the vegetative cell.

Figure 4. A summary diagram of metabolomic changes in S. pombe cells at the onset of nitrogen starvation.

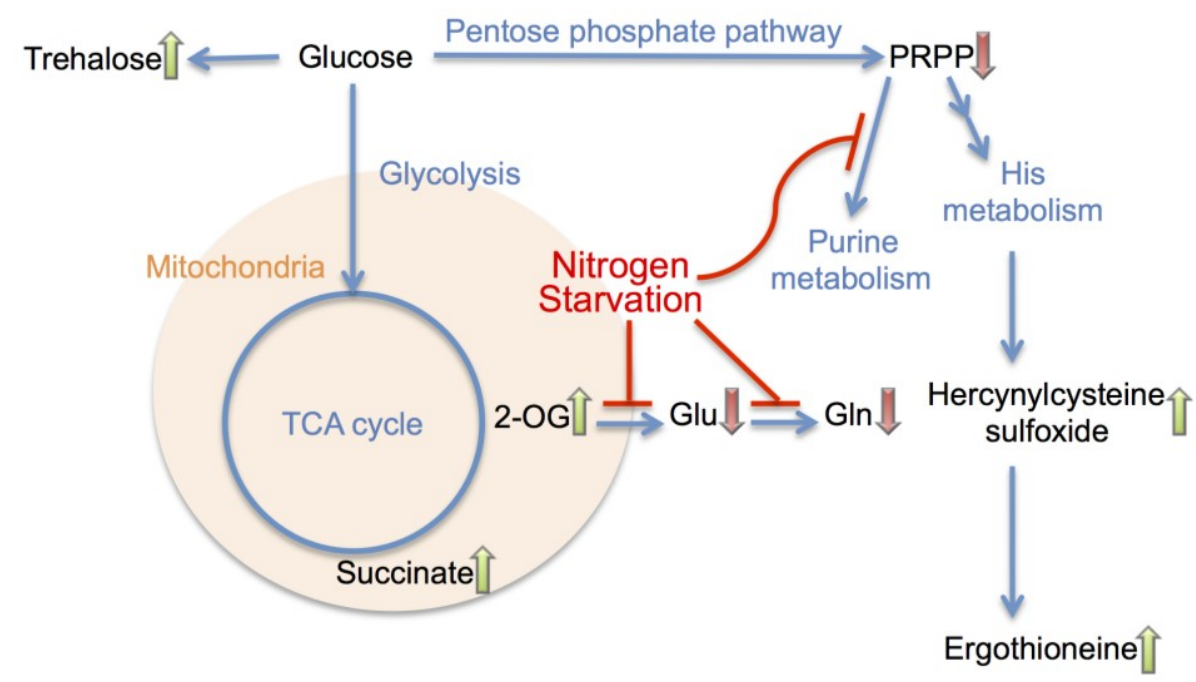

In the previously published transcriptomic and proteomic analyses, a significant overlap between stress response and N-starvation response was evident [5]. At the level of metabolome, this was reflected by the accumulation of trehalose and ergothioneine, both of which have protective functions. However, as we previously observed a similar accumulation of trehalose and ergothioneine under glucose starvation [19], such response seems to be rather non-specific. The rapid and steep accumulation of $2 \mathrm{OG}$, on the other hand, seems to be specific to N-starvation and can be considered a promising biomarker of this condition. It is worth mentioning that the rapidly accumulated compounds (more than 5-fold in $15 \mathrm{~min}$ ) such as 2OG, succinate and trehalose, do not contain nitrogen.

The depletion of nitrogen source led immediately to the reduction in purine biosynthesis intermediates. Among these intermediates was AICAR, a well-known activator for the cellular energy homeostasis regulator AMPK. AICAR is also a strong inhibitor of autophagy [29,30]. Since autophagy is known to be active after N-starvation [31], the decrease in AICAR level could act as a trigger for autophagy. The level of most free amino acids started decreasing only after $15 \mathrm{~min}$ from $\mathrm{N}$-starvation, possibly due to the depletion of residual nitrogen source. On the contrary, the observed accumulation 
of several methylated amino acids brings up an interesting hypothesis that these metabolites might play some physiological role in the adaptation to $\mathrm{N}$-starvation. The simplest explanation could be that these compounds represent a way to stock nitrogen at the metabolic level.

Our key results (increase in $2 \mathrm{OG}$ and trehalose, decrease in amino acids) are consistent with the nitrogen starvation metabolome results in S. cerevisiae [32]. On the other hand, the quick depletion of purine intermediates such as AICAR was not previously described. Also, ergothioneine accumulation seems to be specific to $S$. pombe, as budding yeast does not produce this compound.

Taken from a wider perspective, the metabolites described in this study shed some light on the immediate - and otherwise unnoticeable - intracellular response to N-starvation. In the future, monitoring the levels of these compounds can provide new insights when diagnosing S. pombe strains that show defects in entry to, maintenance of, or exit from N-starvation.

\section{Acknowledgments}

This study was funded by the Okinawa Institute of Science and Technology Promotion Corporation (until October 2011) and Okinawa Institute of Science and Technology Graduate University (from November 2011).

\section{Conflicts of Interest}

The authors declare no conflict of interest.

\section{Supplementary Material}

Supplementary materials can be accessed at: http:/www.mdpi.com/2218-1989/3/4/1118/s1.

The raw LC-MS data in mzML format was submitted to the MetaboLights repository (URL: http://www.ebi.ac.uk/metabolights) under accession number MTBLS67.

\section{References}

1. Yanagida, M. Cellular quiescence: Are controlling genes conserved? Trends Cell Biol. 2009, 19, 705-715.

2. Shimanuki, M.; Chung, S.Y.; Chikashige, Y.; Kawasaki, Y.; Uehara, L.; Tsutsumi, C.; Hatanaka, M.; Hiraoka, Y.; Nagao, K.; Yanagida, M. Two-step, extensive alterations in the transcriptome from g0 arrest to cell division in schizosaccharomyces pombe. Genes Cells 2007, 12, 677-692.

3. Nurse, P.; Bissett, Y. Gene required in $\mathrm{g} 1$ for commitment to cell cycle and in $\mathrm{g} 2$ for control of mitosis in fission yeast. Nature 1981, 292, 558-560.

4. Yanagida, M.; Ikai, N.; Shimanuki, M.; Sajiki, K. Nutrient limitations alter cell division control and chromosome segregation through growth-related kinases and phosphatases. Philos. Trans. R. Soc. Lond. Series B Biol. Sci. 2011, 366, 3508-3520.

5. Marguerat, S.; Schmidt, A.; Codlin, S.; Chen, W.; Aebersold, R.; Bahler, J. Quantitative analysis of fission yeast transcriptomes and proteomes in proliferating and quiescent cells. Cell 2012, 151, $671-683$. 
6. Costello, G.; Rodgers, L.; Beach, D. Fission yeast enters the stationary phase g0 state from either mitotic g1 or g2. Curr. Genet. 1986, 11, 119-125.

7. Su, S.S.; Tanaka, Y.; Samejima, I.; Tanaka, K.; Yanagida, M. A nitrogen starvation-induced dormant $\mathrm{g} 0$ state in fission yeast: The establishment from uncommitted g1 state and its delay for return to proliferation. J. Cell Sci. 1996, 109, 1347-1357.

8. Mata, J.; Bahler, J. Global roles of Ste11p, cell type, and pheromone in the control of gene expression during early sexual differentiation in fission yeast. Proc. Natl. Acad. Sci. USA 2006, 103, 15517-15522.

9. Kristell, C.; Westholm, J.O.; Olsson, I.; Ronne, H.; Komorowski, J.; Bjerling, P. Nitrogen depletion in the fission yeast schizosaccharomyces pombe causes nucleosome loss in both promoters and coding regions of activated genes. Genome Res. 2010, 20, 361-371.

10. Sajiki, K.; Hatanaka, M.; Nakamura, T.; Takeda, K.; Shimanuki, M.; Yoshida, T.; Hanyu, Y.; Hayashi, T.; Nakaseko, Y.; Yanagida, M. Genetic control of cellular quiescence in S. pombe. J. Cell Sci. 2009, 122, 1418-1429.

11. Alfredsson-Timmins, J.; Kristell, C.; Henningson, F.; Lyckman, S.; Bjerling, P. Reorganization of chromatin is an early response to nitrogen starvation in schizosaccharomyces pombe. Chromosoma 2009, 118, 99-112.

12. De Hoon, M.J.; Imoto, S.; Nolan, J.; Miyano, S. Open source clustering software. Bioinformatics 2004, 20, 1453-1454.

13. Saldanha, A.J. Java treeview-extensible visualization of microarray data. Bioinformatics 2004, 20, 3246-3248.

14. Pluskal, T.; Castillo, S.; Villar-Briones, A.; Oresic, M. MZmine 2: Modular framework for processing, visualizing, and analyzing mass spectrometry-based molecular profile data. BMC Bioinforma. 2010, 11, doi:10.1186/1471-2105-11-395.

15. Klosinska, M.M.; Crutchfield, C.A.; Bradley, P.H.; Rabinowitz, J.D.; Broach, J.R. Yeast cells can access distinct quiescent states. Genes Dev. 2011, 25, 336-349.

16. Elbein, A.D.; Pan, Y.T.; Pastuszak, I.; Carroll, D. New insights on trehalose: A multifunctional molecule. Glycobiology 2003, 13, 17R-27R.

17. Chen, D.; Toone, W.M.; Mata, J.; Lyne, R.; Burns, G.; Kivinen, K.; Brazma, A.; Jones, N.; Bahler, J. Global transcriptional responses of fission yeast to environmental stress. Mol. Biol. Cell 2003, 14, 214-229.

18. Pluskal, T.; Nakamura, T.; Villar-Briones, A.; Yanagida, M. Metabolic profiling of the fission yeast $S$. pombe: Quantification of compounds under different temperatures and genetic perturbation. Mol. Biosyst. 2010, 6, 182-198.

19. Pluskal, T.; Hayashi, T.; Saitoh, S.; Fujisawa, A.; Yanagida, M. Specific biomarkers for stochastic division patterns and starvation-induced quiescence under limited glucose levels in fission yeast. FEBS J. 2011, 278, 1299-1315.

20. Perysinakis, A.; Kinghorn, J.R.; Drainas, C. Glutamine synthetase/glutamate synthase ammonium-assimilating pathway in schizosaccharomyces pombe. Curr. Microbiol. 1995, 30, 367-372. 
21. Clifton, I.J.; McDonough, M.A.; Ehrismann, D.; Kershaw, N.J.; Granatino, N.; Schofield, C.J. Structural studies on 2-oxoglutarate oxygenases and related double-stranded beta-helix fold proteins. J. Inorg. Biochem. 2006, 100, 644-669.

22. Zhang, Y.; Morar, M.; Ealick, S.E. Structural biology of the purine biosynthetic pathway. Cell. Mol. Life Sci. 2008, 65, 3699-3724.

23. Yang, P.; Du, H.; Hoffman, C.S.; Marcus, S. The phospholipase b homolog plb1 is a mediator of osmotic stress response and of nutrient-dependent repression of sexual differentiation in the fission yeast Schizosaccharomyces pombe. Mol. Genet. Genomics 2003, 269, 116-125.

24. Cheah, I.K.; Halliwell, B. Ergothioneine, antioxidant potential, physiological function and role in disease. Biochim. Biophys. Acta (BBA)-Mol. Basis Dis. 2012, 1822, 784-793.

25. Bello, M.H.; Barrera-Perez, V.; Morin, D.; Epstein, L. The neurospora crassa mutant ncdeltaegt-1 identifies an ergothioneine biosynthetic gene and demonstrates that ergothioneine enhances conidial survival and protects against peroxide toxicity during conidial germination. Fungal Genet. Biol. 2012, 49, 160-172.

26. Soga, T.; Baran, R.; Suematsu, M.; Ueno, Y.; Ikeda, S.; Sakurakawa, T.; Kakazu, Y.; Ishikawa, T.; Robert, M.; Nishioka, T.; et al. Differential metabolomics reveals ophthalmic acid as an oxidative stress biomarker indicating hepatic glutathione consumption. J. Biol. Chem. 2006, $281,16768-16776$.

27. Gutz, H.; Heslet, H.; Leupold, U.; Loprieno, N. Schizosaccharomyces pombe. In Handbook of Genetics; Plenum Press: New York, NY, USA, 1974.

28. Mitchison, J.M. Physiological and cytological methods for Schizosaccharomyces pombe. Method Cell Physiol. 1970, 4, 131-165.

29. Meley, D.; Bauvy, C.; Houben-Weerts, J.H.; Dubbelhuis, P.F.; Helmond, M.T.; Codogno, P.; Meijer, A.J. Amp-activated protein kinase and the regulation of autophagic proteolysis. J. Biol. Chem. 2006, 281, 34870-34879.

30. Samari, H.R.; Seglen, P.O. Inhibition of hepatocytic autophagy by adenosine, aminoimidazole-4carboxamide riboside, and n6-mercaptopurine riboside. Evidence for involvement of amp-activated protein kinase. J. Biol. Chem. 1998, 273, 23758-23763.

31. Kohda, T.A.; Tanaka, K.; Konomi, M.; Sato, M.; Osumi, M.; Yamamoto, M. Fission yeast autophagy induced by nitrogen starvation generates a nitrogen source that drives adaptation processes. Genes Cells 2007, 12, 155-170.

32. Boer, V.M.; Crutchfield, C.A.; Bradley, P.H.; Botstein, D.; Rabinowitz, J.D. Growth-limiting intracellular metabolites in yeast growing under diverse nutrient limitations. Mol. Biol. Cell 2010, $21,198-211$.

(C) 2013 by the authors; licensee MDPI, Basel, Switzerland. This article is an open access article distributed under the terms and conditions of the Creative Commons Attribution license (http://creativecommons.org/licenses/by/3.0/). 